\title{
INFLUENCE OF MULTIPLE TRAPPING PHENOMENA ON THE APPLICATIONS CRITERIA OF $\mathrm{ZnO}^{-\mathrm{Bi}_{2}} \mathrm{O}_{3}$-BASED VARISTORS
}

\author{
MOHAMMAD A. ALIM \\ Hubbell Incorporated, The Ohio Brass Company, 8711 Wadsworth Road, Wadsworth, Ohio 44281 \\ (Received January 31, 1994; in final form February 22, 1994)
}

The performance characteristics of the $\mathrm{ZnO}-\mathrm{Bi}_{2} \mathrm{O}_{3}$-based varistors can be assessed to meet the applications criteria on the basis of a systematic evaluation corresponding to various experimental conditions. This evaluation process employs the lumped parameter/complex plane analysis technique for the ac small-signal immittance data. An overall behavior of these devices is attributed to the microstructural effects via controlled grain-size and its distribution, existence of phases, carrier density in the grains, trapping states, and their role within the electrical thickness across the grain-boundary electrical barrier, etc. These factors are strongly dictated by the additives to $\mathrm{ZnO}$ in conjunction with the processing variables. Multiple device functions can result when a single set from a variety of processing parameters is chosen, provided the starting chemistry or composition/formulation remain invariant. The factors related to materials' history, composition recipe, and combined processing methods influence or modify relative magnitudes of the constituting elements of each trapping relaxation. Thus, these magnitudes either increase or decrease the visibility without distorting the devices' generic dielectric behavior. An identical set of experiments contributes to distinguish a good varistor performance over a poor response for surge arrester (i.e., suppressor/absorber) applications in the power systems' transient protection.

\section{INTRODUCTION}

The $\mathrm{ZnO}-\mathrm{Bi}_{2} \mathrm{O}_{3}$ based highly nonlinear devices are commonly known as metal oxide varistors $^{1}$ (MOVs). The electrical surge arresters are based upon the MOV blocks, arranged in a stack or in several electrically series-connected stacks in the valve section between the top and bottom terminals ${ }^{2}$ inside an insulating (ceramic porcelain or polymer rubber) housing. These are used extensively to protect electrical equipment and increase voltage stability. ${ }^{1-3}$ Also, reliability of electrical power distribution is achieved via transient protection against lightning, switching pulse, temporary overvoltage (TOV), etc. The MOVs are primarily composed of $\mathrm{ZnO}$ with small additives of $\mathrm{Bi}_{2} \mathrm{O}_{3}$ and other oxide constituents. ${ }^{1-4}$ These additives control each electrically active grain-boundary (GB) electrical barrier, physically existing within the electrical length (i.e., electric field falling region), resulting from the charge trapping processes. ${ }^{1.5-9}$ In general, the high resistivity exhibited by MOVs at low electric stress levels is derived from these grain boundaries, which are strongly insulating with respect to the n-type $\mathrm{ZnO}$ grains they surround. An overall electrical performance (including protective characteristics) of the device represents lumped behavior of the microstructural operative paths between the electrode terminals. 
A lumped single electrical barrier is the effective representation of the microstructural network consisting of the " $m$ " junctions in parallel with " $n$ " junctions in series between the electrodes. ${ }^{5.10}$

Essentially, the performance characteristics of a MOV are controlled by the presence and nature of the defect states and interactions with the carriers via trapping-detrapping across the GB barriers. The role of other phases at the GB interfaces and tri-/tetra-grain corner regions or junctions are often not clearly understood. Their importance in the microstructure via accelerated aging or lifetest at elevated temperatures stressing at MCOV (maximum continuous operating voltage), thermal properties (i.e., thermal diffusivity/conductivity, heat generation/ dissipation, specific heat, etc.), etc., are not necessary and thus, neglected. These phases may constitute a passive role (such as: heat-flow/propagation, energy-handling, thermal runaway, etc.) during the severe conduction processes, without being destroyed, between the electrodes under specific experimental circumstances. This, however, has not yet been addressed adequately in the literature. The microstructural features and their reproducibility with a specific chemistry or composition/ formulation, and multiple processing routes provide the resulting performance characteristics of the device. A minor sequential or simultaneous variation either in composition recipe or processing route results in more than one type of varistor materials. This implies that a single starting MOV composition recipe can yield several types of devices as the end-product, possessing a variety of performance characteristics that depend on the routes for a combined set of processing variables. Therefore, an optimization of the formulation with a desired set of electrical performance characteristics using a fixed processing route is achievable. As a next step, a non-destructive assessment on the merit of a MOV valve block is desirable before stacking in the arrester assembly.

Several published documents $\mathrm{s}^{5-9}$ reveal the presence of simultaneously competing trapping phenomena and their role in the MOVs' function are the issues in determining time-dependent applications as surge arresters. From a commercial viewpoint, a well-formed (exhibiting or possessing good performance characteristics for applications) varistor possesses desired properties for such applications. A distinction between a good performance and a poor response of these devices is possible when they are examined under identical experimental conditions employing immittance (impedance or admittance) spectroscopy. This distinction is based upon the acquisition of the ac small-signal immittance data and subsequent utilization of the lumped parameter/complex plane analysis (LP/CPA) technique.

The LP/CPA technique is a viable non-destructive spectroscopic approach s-7,10-12 $^{-12}$ for the immittance data to determine the nature of the microstructural electrical path(s) between the electrodes. This technique provides a basis for understanding the character of a multi-component/phase heterogeneous device system, such as MOV, resolving extraneous contributions to the terminal immittance operative within it. ${ }^{5-7,10-12}$ These contributions are unraveled in the frequency domain. For the MOVs, wide dispersion in terminal immittance with the ac frequency encompasses large capacitance, often regardless of the contributing elements to it, for the same range of measurement frequency. The resulting performance character- 
istics of these MOVs depend on the nature of this dispersion. An effective judgement on the prediction of an overall performance is possible from this dispersion.

In this paper, a demarcation between a good and a poor electrical performance of the MOVs, using the LP/CPA technique resolving multiple competing phenomena, is postulated. This demarcation is effective, and primarily based on the combined trapping response possessing interrelationships among each of these traps. The origin of these simultaneously operative trapping phenomena remains complicated, and attributes to several plausible simple charge states, complex radicals, and their combination.

\section{IMMITTANCE-FREQUENCY DISPERSION}

The two-terminal immittance data of the MOVs, acquired over a frequency range $10^{-2} \mathrm{~Hz} \leqslant \mathrm{f} \leqslant 10^{9} \mathrm{~Hz}$, reveal multiple relaxations $s^{5-7,11}$ and a resonance phenomenon ${ }^{1.5-7.11}$ in the complex capacitance $\left(C^{*}=C^{\prime}-j C^{\prime \prime}=C_{p}-j G_{p} / \omega\right.$; where $C^{\prime}=C_{p}=$ terminal parallel capacitance, $C^{\prime \prime}=G_{p} / \omega, G_{p}=$ terminal parallel conductance, $\omega=2 \pi f$, and $\mathrm{j}=\sqrt{-1}$ ) plane. A generic representation of the immittance data at near room-temperature is provided in Figure 1. In this plot, three distinct relaxations [denoted as $\tau_{2}\left(=\mathrm{R}_{2} \mathrm{C}_{2}\right), \tau_{3}\left(=\mathrm{R}_{3} \mathrm{C}_{3}\right)$, and $\tau_{4}\left(=\mathrm{R}_{4} \mathrm{C}_{4}\right)$ ], a low-frequency distortion $\left(\mathrm{C}_{1}\right.$ or corresponding polarization related $\tau_{1}$ relaxation), and a barrier layer capacitance $\left(\mathrm{C}_{\mathrm{BL}}=\mathrm{C}_{5}+\mathrm{C}_{6}\right)$ including the dielectric contribution of the lumped grains $\left(\mathrm{C}_{6}=\mathrm{C}_{\mathrm{ZnO}}\right)$ are evident. These six delineated capacitive elements, denoted as $\mathrm{C}_{1}$ through $\mathrm{C}_{6}$, represent static (i.e., under dc condition) capacitance $^{5,6.10 .12}$ when lumped together. The nomenclature of each capacitive element is cited in references 5 and 6 . The relative contribution of each element can reveal experimental dependence under a given set of conditions.

The capacitances $\mathrm{C}_{5}$ and $\mathrm{C}_{6}$ (or combined $\mathrm{C}_{\mathrm{BL}}$ ), in general, are nearly identical for all devices (unless otherwise the purpose of application is drastically different), and their lumped behavior is geometric. This is because of nearly uniform spatial carrier density in the $\mathrm{ZnO}$ grains and defect states ensuing identical electrical thickness (i.e., electric field falling depletion region) across the GB. Thus, the geometric nature of $\mathrm{C}_{\mathrm{BL}}$ causes either no or less impact on the static capacitance (i.e., $\sum_{i=1}^{6} C_{i}$ ) with ac the frequency.

The uniformity in the spatial carrier density in the $\mathrm{ZnO}$ grains is evidenced by the frequency-independent Mott-Schottky (i.e., $1 / \mathrm{C}_{\mathrm{BL}}^{2}$ versus applied dc voltage) straight line and successfully demonstrated in reference 6 . The net defect states within the electrical thickness are likely to be primarily dependent on the grainsize and phase distribution in the microstructure. The origin of these defects are widely speculated ${ }^{1-3}$ but often seem to stand alone lacking firm support. In this multi-component/phase heterogeneous device, the nature of these defects is likely to be more complex than relating them to the singular defect status of the additive species. Nevertheless, these defects within the electrical thickness presumably controls $\mathrm{C}_{1}+\mathrm{C}_{2}+\mathrm{C}_{3}+\mathrm{C}_{4}$ (or $\Sigma_{\mathrm{i}=1}^{4} \mathrm{C}_{\mathrm{i}}$ ). Thus, the capacitances $\mathrm{C}_{1}$ through $\mathrm{C}_{4}$, attributed to trapping, ${ }^{5,6}$ essentially dominate the overall static dielectric behavior 


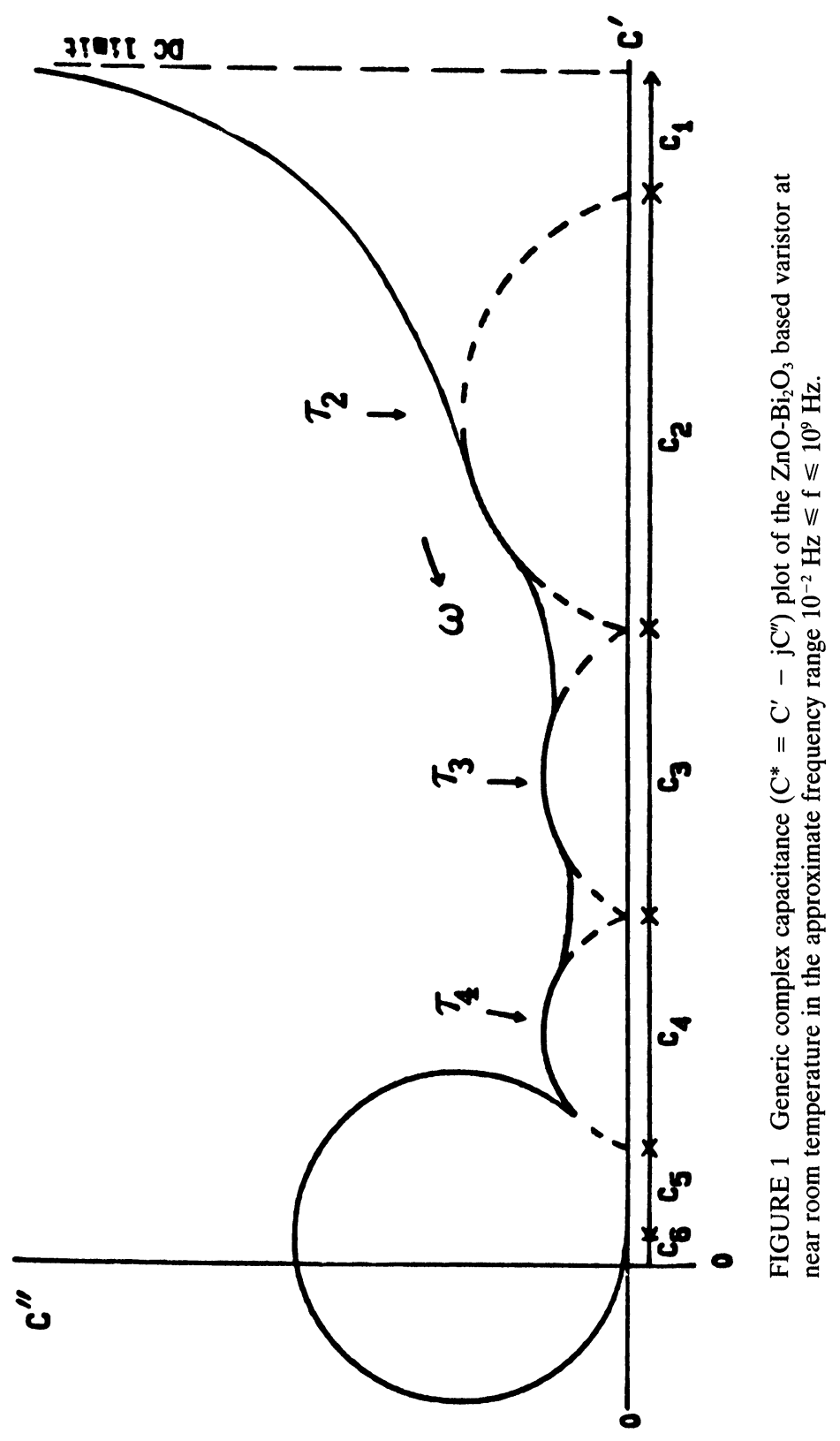


of the devices. In general, the aforementioned distinguishable behavior in the frequency domain is a characteristic of the well-formed varistor composites, regardless of manufacturer's materials' history and processing methods. These factors influence relative magnitudes, and increase or decrease the visibility of all the constituting parameters (i.e., $\mathrm{R}_{\mathrm{i}}$ and $\mathrm{C}_{\mathrm{i}}$ for each $\tau_{\mathrm{i}}$ ) without eliminating or distorting generic response of this dielectric composite.

Each distinct relaxation (i.e., $\tau_{2}, \tau_{3}$, and $\tau_{4}$ ) exhibit depressed semicircle ${ }^{5-7}$ in the $C^{*}$-plane (Figure 1). An example of each of these relaxations can be seen in references 5 and 6 . The $\tau_{3}$ semicircular relaxation is common to all well-formed devices and attributed to an intrinsic response. Often, $\tau_{2}$ relaxation is not straightforward and found to be heavily distorted similar to the $\tau_{1}$ relaxation. In such cases, a wide range in measurement frequencies (including low frequencies) can delineate the total value for the $\sum_{\mathrm{i}=1}^{6} \mathrm{C}_{\mathrm{i}}$. For this purpose, these data must be analyzed in the impedance $\left(Z^{*}\right)$ plane to obtain a single semicircular relaxation. ${ }^{5,6,10,12}$ This semicircular relaxation is usually depressed, and the corresponding relaxation time $\left(\tau_{\mathrm{im}}\right)$ originates from its chord and capacitance at dc condition (i.e., $\sum_{\mathrm{i}=1}^{6} \mathrm{C}_{\mathrm{i}}$ ). An example of the cross examination between the $\mathrm{C}^{*}$ - and $\mathrm{Z}^{*}$-plane representations is provided in references 5 and 6 . In this way, $C_{1}+C_{2}$ can be obtained as a lumped single quantity. The chord in the $Z^{*}$-plane semicircular relaxation essentially provides an approximate dc resistance $\left(R_{d c}\right)$ value of the device. This approximation is based upon a finite ultra-small ${ }^{5-7.12}$ (as an example: $10 \Omega$ is much smaller than $10^{5} \Omega$ or several orders greater) left-intercept (related to the lumped $\mathrm{ZnO}$ grains) of the semicircle.

At high frequencies (usually $10^{6} \leqslant \mathrm{f} \leqslant 10^{9} \mathrm{~Hz}$ ), a resonance phenomenon emerges as a circle in the $C^{*}$-plane (Figure 1 ), possessing negative values of the terminal parallel capacitance. This resonance behavior is complex ${ }^{1,5,6,11}$ and attributed to the combined external electrode-lead configuration, built-in contact inductance of the sample holder, and possible piezoelectric grain resonance phenomenon. The variation in lead length and sample thickness with a fixed electrode area influences the onset of this behavior with respect to the measurement frequency. Three types of resonance events ${ }^{11}$ are observed which depend on the nature of the overall lumped GB response in conjunction with electrode configuration while approaching high frequencies. A detailed description on these resonance events is provided in reference 11 . Only one type of resonance event, identical to Figure 1, allows a distinct $\tau_{4}$ relaxation yielding $\mathrm{C}_{5}$ and $\mathrm{C}_{6}$. The other two events exhibit a distinct $\tau_{3}$ relaxation with the masking of $\tau_{4}$ relaxation. ${ }^{1.5-7.11}$

\section{GOOD AND POOR VARISTOR RESPONSE}

Since $\mathrm{C}_{1}$ through $\mathrm{C}_{4}$ exhibit a strong frequency dependence, it is reasonable to distinguish extreme kinds of devices with respect to their magnitudes. In fact, these capacitances produce complicated and deceptive or unlimited frequency-dependent Mott-Schottky straight lines. ${ }^{6}$ Often, no Mott-Schottky plot is possible below 100 $\mathrm{Hz}$ due to noise and operative polarization processes within the device. Such a situation leads to two distinct cases, observed for all normalized (i.e., considering 
identical geometry or dimension) configuration of the MOV samples at a specific (i.e., arbitrarily chosen) terminal frequency $\left(f_{p}\right)$, before approaching the resonance phenomenon. These include terminal parallel capacitance $\left(C_{p}\right)$, which can be distinguished as: $(a)$ large and $(b)$ small. Extending $C_{p}$ to the dc condition, assuming nearly constant $\mathrm{C}_{\mathrm{BL}}$, this observation provides:

$$
\sum_{i=1}^{6} C_{i}=\text { large, and } \sum_{i=1}^{6} C_{i}=\text { small. }
$$

The quantities large and small are absolutely dependent on the devices' composition recipe and processing variables. Figure 1 is the key representation to distinguish the quantities large and small in terms of the terminal (i.e., at $\mathrm{f}=$ measurement frequency) and/or dc (i.e., $\mathrm{f}=0 \mathrm{~Hz}$ ) condition.

In order to distinguish between two unknown MOV samples, the foregoing situation at dc condition becomes critical. Based on general observation, for small $\mathrm{C}_{\mathrm{p}}$ or $\sum_{\mathrm{i}=1}^{6} \mathrm{C}_{\mathrm{i}}$ a device results in an acceptable performance while for large yields poor response. Upon inspecting the values of $\mathrm{C}_{\mathrm{i}}(\mathrm{i}=1$ through 6$)$ a tentative scrutiny is possible. This implies a clear distinction among the devices and ultimately leads to a demarcation between a good and a poor response. Such a decision is purely based on a systematic trend of the ac electrical response for these devices. Thus, these devices can be classified in a sequential order with respect to the composition recipe and processing history. The exact reason for such empirical establishment between the nature of the dispersion in terminal immittance as a function of frequency and performance characteristics is not known at this time. However, this classification is attributed to the trap-related defect charge states causing extraneous $\mathrm{C}_{1}$ through $\mathrm{C}_{4}$, and thereby sustaining electrical barriers across the grain-boundaries.

In the event terminal parallel capacitance corresponding to a specific frequency or at dc condition $\left(\sum_{i=1}^{6} C_{i}\right)$ is large, a MOV has the following features:

1. either not or less durable (i.e., accelerated aging or life-time) at MCOV with the possibility of undergoing a thermal runaway in the temperature range $115^{\circ} \mathrm{C} \leqslant \mathbf{T} \leqslant 140^{\circ} \mathrm{C}$;

2. poor heat-dissipation and/or energy-handling capability leading to a failure at high-current impulses and energy-handling tests;

3. high or increased watts-loss and capacitive-current with low-leakage resistance (in the ohmic region);

4. likely to possess higher degree of meta-stability in electrical barriers and fluctuation/drift in leakage current (or polarization effect) in the temperature range $20^{\circ} \mathrm{C} \leqslant \mathrm{T} \leqslant 90^{\circ} \mathrm{C}$;

5. generally higher degree of inhomogeneity in electrical path(s) within the microstructure associated with the common (intrinsic) trapping loss parameter (denoted as $\tau_{3}$ relaxation in references 5 through 7 ) and large perturbation in low-frequency (usually $\mathrm{f} \leqslant 10^{3} \mathrm{~Hz}$ ) related interfacial traps (i.e., $\tau_{1}$ and $\tau_{2}$ as referred in references 5 and 6 ) influenced by the polarization effect (or drift at quasi-equilibrium conditions); 
6. barely or not likely to meet surge suppressor design requirements even combined with the supplementary active/passive element(s) in the arrester assembly;

7. spectra of the dissipation factor (i.e., $\tan \delta$ ) usually large with the dispersion in frequency in the range $10 \mathrm{~Hz} \leqslant \mathrm{f} \leqslant 10^{7} \mathrm{~Hz}$, and corresponding typical value ranges $2.5^{\circ} \leqslant \delta \leqslant 6^{\circ}$ (i.e., $-87.5^{\circ} \leqslant \delta \leqslant-84^{\circ}$ );

8. less sharp transition between the intrinsic $\left(\tau_{3}\right)$ and low-frequency related $\tau_{2}$ trapping phenomena (i.e., larger or broader overlapped region between these two relaxations);

9. intrinsic trapping time constant $\left(\tau_{3}\right)$ typically ranges between $1 \mu \mathrm{s}$ and $2 \mu \mathrm{s}$ (sometimes out of this range), where the constituting elements $R_{3}$ is low (typically $\sim 10^{3} \Omega \leqslant R \leqslant \approx 4 \times 10^{4} \Omega$ ) and $C_{3}$ is high (usually same order as the terminal parallel capacitance, i.e., on the order of $\mathrm{pF}$ to satisfy the value of $\tau_{3}$ );

10. the depression angle parameter, $\mathrm{h}_{3}\left(=2 \theta_{3} / \pi\right.$, where $\theta_{3}$ is the depression angle obtained in the $\mathrm{C}^{*}$-plane ${ }^{5-7}$ for the common $\tau_{3}$ semicircular relaxation), usually corresponds to greater than 0.4 or $\theta_{3} \geqslant 36^{\circ}$;

11. the depression angle parameter, $\mathrm{h}_{\mathrm{im}}\left(=2 \theta_{\mathrm{im}} / \pi\right.$, where $\theta_{\mathrm{im}}$ is the depression angle obtained in the $\mathrm{Z}^{*}$-plane $\mathrm{e}^{6.10 .12}$ for the total behavior of the device), usually corresponds to greater than 0.2 or $\theta_{\text {im }} \geqslant 18^{\circ}$; and

12. visible noise at $f \leqslant 500 \mathrm{~Hz}$ and extremely short-range straight line in the Mott-Schottky plot. ${ }^{6}$

In the event terminal parallel capacitance corresponding to a specific frequency or at dc condition $\left(\sum_{i=1}^{6} C_{i}\right)$ is small, a MOV has the following features:

1. generally durable at MCOV and slight possibility of undergoing a thermal runaway in the temperature range $115^{\circ} \mathrm{C} \leqslant \mathbf{T} \leqslant 140^{\circ} \mathrm{C}$;

2 . better heat dissipation and energy-handling exhibiting enhanced withstanding capability at high current impulses and energy-handling tests leading to either less or no failure;

3. usually low or decreased watts-loss and capacitive-current with reasonably low leakage current (i.e., high leakage resistance in the ohmic region);

4. possess lesser degree of meta-stability in electrical barriers with the possibility of sub-ohmic response (not detrimental) in the leakage region;

5. lesser degree of inhomogeneity in electrical path(s) associated with the common (intrinsic) trapping loss parameter, and suppressed perturbation in low-frequency related traps with minimal polarization effect or drift at quasi-equilibrium conditions;

6. generally meet surge suppressor design requirements with high success rates;

7. spectra of dissipation factor usually small with the dispersion in frequency in the range $10 \mathrm{~Hz} \leqslant \mathrm{f} \leqslant 10^{7} \mathrm{~Hz}$, and corresponding typical value ranges $0.3^{\circ} \leqslant \delta \leqslant 3^{\circ}$ (i.e., $-89.7^{\circ} \leqslant \delta \leqslant-87^{\circ}$, the overlapping with the poor devices is distinguished via other factors, such as: $\theta_{3}, \theta_{\text {im }}$, low-frequency dispersion, polarization effect, etc.);

8. generally possess a sharp transition between the intrinsic $\left(\tau_{3}\right)$ and low-fre- 
quency related $\tau_{2}$ trapping phenomena (i.e., smaller overlapped region between these two relaxations);

9. intrinsic trapping time constant $\left(\tau_{3}\right)$ ranging, in general, between $1 \mu$ s and $2 \mu \mathrm{s}$, where $R_{3}$ is high (typically $\sim 10^{4} \leqslant R_{3} \leqslant \sim 10^{5} \Omega$ ) and $C_{3}$ is low (i.e., low-side order in magnitude of the terminal parallel capacitance);

10. the common depression angle parameter, $\mathrm{h}_{3}$, usually corresponds to less than 0.4 (i.e., finite or nonvanishing) or $12^{\circ} \leqslant \theta_{3} \leqslant 36^{\circ}$;

11. the total depression angle parameter, $\mathrm{h}_{\mathrm{im}}$, usually corresponds to less than 0.2 (i.e., finite or non-vanishing) or $10^{\circ} \leqslant \theta_{\text {im }} \leqslant 18^{\circ}$; and

12. reduced level of noise at $\mathrm{f} \leqslant 200 \mathrm{~Hz}$ and moderately short-range straight line in the Mott-Schottky plot. ${ }^{6}$

The proof of the foregoing classified features remains empirical in nature, and absolutely based upon a systematic series of sequential and concurrent evaluation of numerous MOV samples possessing various processing history. The information, thus summarized, is useful, and often, the origin of each classified feature is not addressed in the literature.

\section{CONCLUSIONS}

The ac small-signal immittance data can effectively utilize the lumped parameter/ complex plane analysis (LP/CPA) technique to distinguish resulting electrical performance characteristics of the $\mathrm{ZnO}-\mathrm{Bi}_{2} \mathrm{O}_{3}$-based varistors. This approach employs a less-expensive and conveniently non-destructive measurement process in an efficient manner. The net multiple trapping response, regardless of their semicircular distortions in the $C^{*}$-plane, identified as the relaxation times $\tau_{1}, \tau_{2}, \tau_{3}$, and $\tau_{4}$, is responsible for an overall performance of these devices. This response can be optimized for the contributions of the constituting components to each relaxation (i.e., $\tau_{\mathrm{i}}=\mathrm{R}_{\mathrm{i}} \mathrm{C}_{\mathrm{i}}$ with $\mathrm{i}=1,2,3$, and 4 ) process via the chemistry or formulation/ composition and processing history. A demarcation between a good and a poor varistor behavior, regardless of the composition recipe and processing variables, is established through a systematic evaluation using the LP/CPA technique.

\section{ACKNOWLEDGMENT}

It is author's pleasure to thank Ms. Sanjida Khanam for her interest and intellectual support.

\section{REFERENCES}

1. (a) L.M. Levinson and H.R. Philipp, "Zinc Oxide Varistors-A Review," Am Ceram Soc. Bull., 65\#4, 639-646 (1986). (b) L.M. Levinson and H.R. Philipp, "High-Frequency and High-Current Studies on Metal Oxide Varistors," J. Appl. Phys., 47, 3116-3121 (1976). (c) L.M. Levinson and H.R. Philipp, "AC Properties of Metal-Oxide Varistors," J. Appl. Phys., 47\#3, 1116-1122 (1976). (d) L.M. Levinson and H.R. Philipp, "The Physics of Metal Oxide Varistors," J. Appl. Phys., 46\#3, 1332-1341 (1975).

2. M.A. Alim; "Passivating Coating for Metal Oxide Varistors," U.S. Patent 5,203,915 (April 20, 1993). (b) T.O. Sokoly and J. Niedzialkowski; "Metal Oxide Varistor Manufacture," U.S. Patent 
4,296,002 (October 20, 1981). (c) J.E. Schroeder and J.F. Rasmussen; "Insulating Coating for Surge Arrester Valve Element," U.S. Patent 4,278,961 (July 14, 1981). (d) L.M. Levinson, H.F. Ellis, and H. Fishman; "Arrester with Graded Capacitance Varistors," U.S. Patent 4,276,578 (June 30, 1981). (e) H.P. Klein and A. Menth; "Ceramic Electrical Resistor with Nonlinear Voltage Characteristic," U.S. Patent 4,127,511 (November 28, 1978). (f) J.S. Kresge and H.E. Fiegel; "Electrical Overvoltage Surge Arrester with a Long Time Constant Valve Section and Series Gap Section," U.S. Patent 3,967,160 (June 29, 1976). (g) H.F. Ellis; "Nonlinear Resistance Surge Arrester Disc Collar and Glass Composition Thereof," U.S. Patent 3,959,543 (May 25, 1976).

3. T.K. Gupta; "Applications of Zinc Oxide Varistors," J. Am. Ceram. Soc., 73(7), 1817-1840 (1990).

4. M. Matsuoka; "Nonohmic Properties of Zinc Oxide Ceramics," Jap. J. Appl. Phys., 10, 736-746 (1971).

5. M.A. Alim; “Admittance-Frequency Response in Zinc Oxide Varistor Ceramics,” J. Am. Ceram. Soc., 72(1), 28-32 (1989).

6. M.A. Alim, M.A. Seitz, and R.W. Hirthe; "Complex Plane Analysis of Trapping Phenomena in Zinc Oxide Varistors," J. Appl. Phys., 63(7), 2337-2345 (1988).

7. L.C. Sletson, M.E. Potter, and M.A. Alim; "Influence of Sintering Temperature on Intrinsic Trapping in Zinc Oxide-Based Varistors," J. Am. Ceram. Soc., 71, 909-913 (1988).

8. (a) J.C. Simpson and J.F. Cordaro; "Defect Clusters in Zinc Oxide," J. Appl. Phys., 67(11), 6760-6763 (1990). (b) Y. Shim and J.F. Cordaro; "Effects of Dopants on the Deep Bulk Levels in the $\mathrm{ZnO}-\mathrm{Bi}_{2} \mathrm{O}_{3}-\mathrm{MnO}_{2}$ System, J. Appl. Phys., 64(8), 3994-3998 (1988). (c) J.C. Simpson and J.F. Cordaro; "Characterization of Deep Levels in Zinc Oxide," J. Appl. Phys., 63(5), 1781-1783 (1988). (d) Y. Shim and J.F. Cordaro; "Admittance Spectroscopy of Polycrystalline $\mathrm{ZnO}^{-\mathrm{Bi}_{2} \mathrm{O}_{3}}$ and ZnO-BaO Systems," J. Am. Ceram. Soc., 71, 184-188 (1988). (e) J.F. Cordaro, Y. Shim, and J.E. May; "Bulk Electron Traps in Zinc Oxide Varistors," J. Appl. Phys., 60, 4186-4190 (1986).

9. J. Tanaka, S.I. Hishita, and H. Okushi; "Deep Levels Near the Grain Boundary in a Zinc Oxide Varistor: Energy Change Due to Electrical Degradation,” J. Am. Ceram. Soc., 73(5), 1425-1428 (1990).

10. M.A. Alim and M.A. Seitz; "Singular Nature of Preferential Conducting Paths at High Electric Fields in ZnO-Based Varistors," J. Am. Ceram. Soc., 71(5), C246-C249 (1988).

11. M.A. Alim; "High-Frequency Terminal Resonance in $\mathrm{ZnO}-\mathrm{Bi}_{2} \mathrm{O}_{3}$ Based Varistors," J. Appl. Phys., 74(9), 5850-5853 (1993).

12. M.A. Alim, M.A. Seitz and R.W. Hirthe, "High-Temperature/Field Alternating Current Behavior of ZnO-Based Varistors," J. Am. Ceram. Soc., 71(1), C52-C55 (1988). 

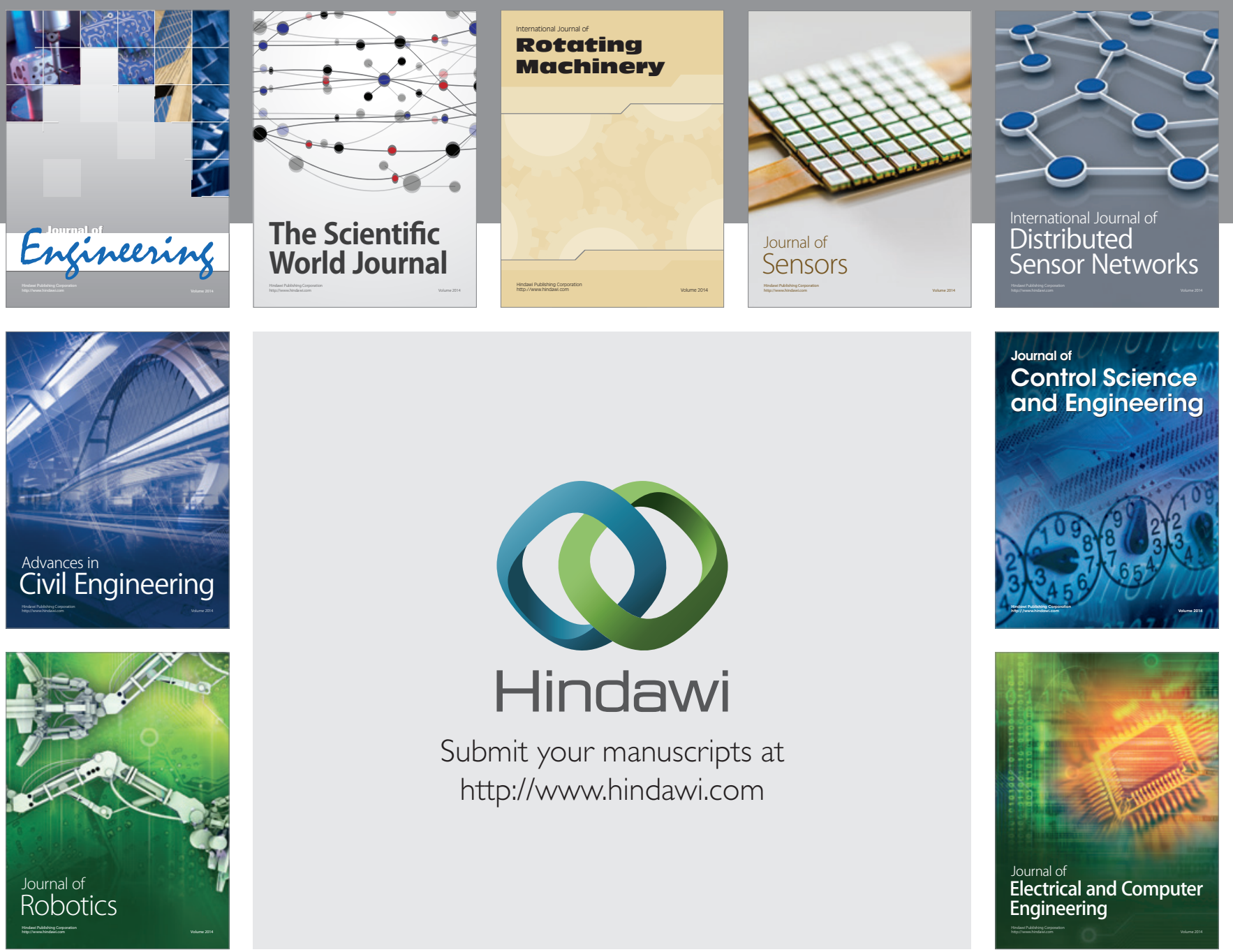

Submit your manuscripts at

http://www.hindawi.com
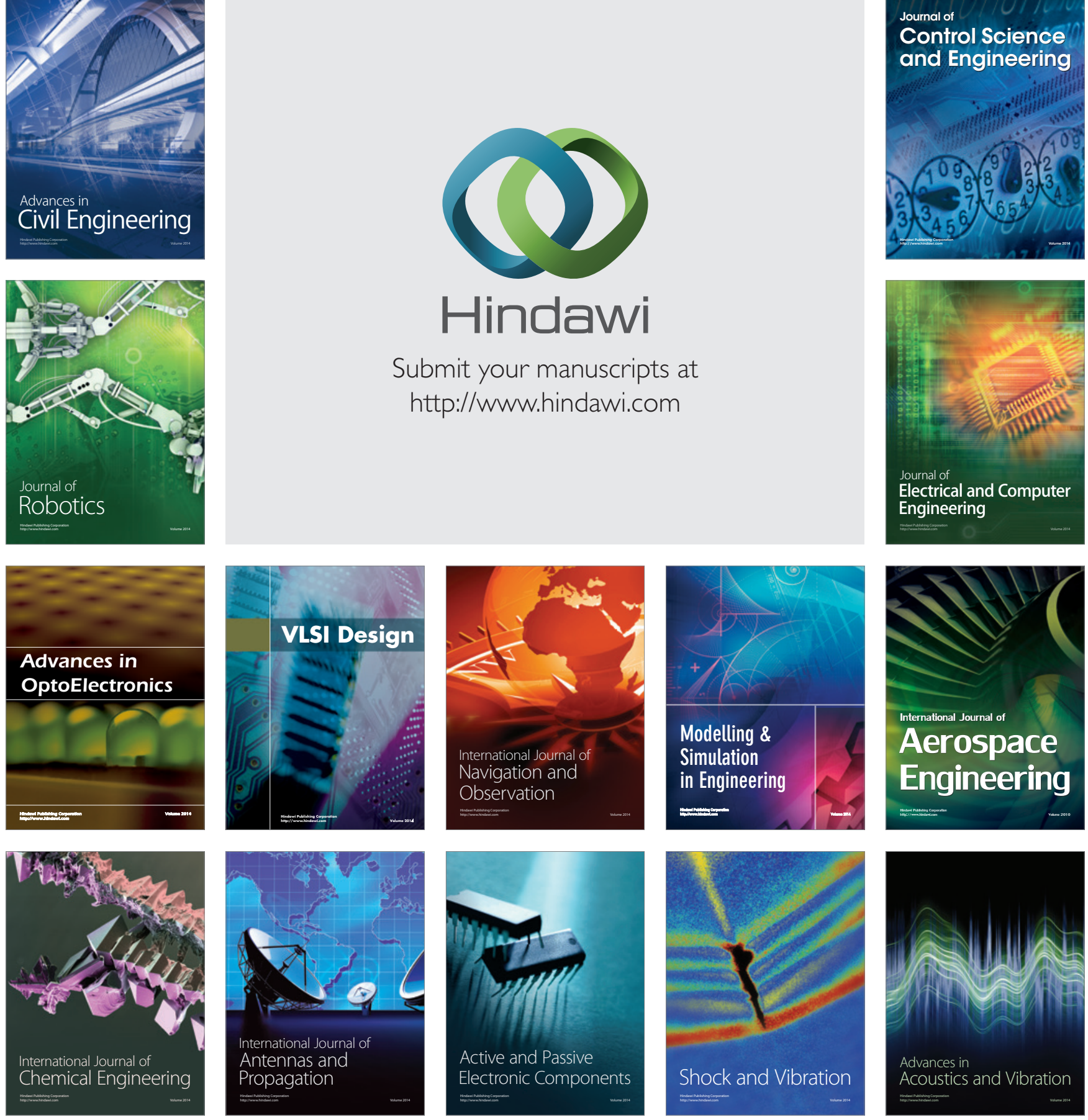